\title{
Asymptotic safety, the Higgs boson mass, and beyond the standard model physics
}

\author{
Jan H. Kwapisz $\oplus^{*}$ \\ Institute of Theoretical Physics, Department of Physics, University of Warsaw, \\ ul. Pasteura 5, 02-093 Warsaw, Poland \\ and Max-Planck-Institut für Gravitationsphysik (Albert-Einstein-Institut), \\ Am Mühlenberg 1D-14476 Potsdam, Germany
}

(Received 27 September 2019; published 2 December 2019)

\begin{abstract}
There are many hints that gravity is asymptotically safe. The inclusion of gravitational corrections can result in the ultraviolet fundamental standard model and constrain the Higgs mass to take the smallest value such that electroweak vacuum is stable. Taking into account the current top quark mass measurements, this value is $m_{H} \approx 130 \mathrm{GeV}$. This article considers the predictions of the Higgs mass in two minimal Beyond standard model scenarios, where the stability is improved. One is the sterile quark axion model, while the other is the $U(1)_{B-L}$ gauge symmetry model introducing a new massive $Z^{\prime}$ gauge boson. The inclusion of $Z^{\prime}$ boson gives the correct prediction for this mass, while inclusion of sterile quark(s) gives only a slight effect. Also a new, gravitational solution to the strong charge parity problem is discussed.
\end{abstract}

DOI: $10.1103 /$ PhysRevD.100.115001

\section{INTRODUCTION}

The couplings of the physical models change with scale, and there are two sources of this scaling. The first, classical scaling is due to canonical dimensionality of the operators. The theory, which is classically scale invariant, possesses dimensionless couplings only in the action. This is indeed the case for the standard model (SM) with zero bare Higgs mass, the so-called conformal standard model [1]. The other source of scaling is caused by the quantum effects, which can spoil classical scale invariance and provide the generation of scale due to radiative corrections. In particular, the Coleman-Weinberg mechanism generates masses in this pattern [2]. In quantum field theories, the change ("running") of couplings with energy scale is described by renormalization group of equations

$$
\mu \frac{\partial}{\partial \mu} g_{i}(\mu)=\beta_{i}\left(\left\{g_{j}\right\}\right)
$$

Such a general equation can have various possible behaviors for $\mu \rightarrow+\infty$, yet only some of them make the theory predictable up to the infinite energies. In the simplest case, the couplings reach the fixed point $\left.\left(\forall_{i} \beta_{i}\left(\left\{g_{j}\right)\right\}\right)=0\right)$ and the running stops, making the theory scale invariant on the

\footnotetext{
*Jan.Kwapisz@fuw.edu.pl
}

Published by the American Physical Society under the terms of the Creative Commons Attribution 4.0 International license. Further distribution of this work must maintain attribution to the author(s) and the published article's title, journal citation, and DOI. Funded by SCOAP ${ }^{3}$. quantum level. However, this is not only possibility, since the coupling can also be attracted to a higher dimensional structure [3], like a limit cycle (see, for example, [4,5] for a limit cycle behavior in $1 / r^{2}$ potential) or a chaotic attractor. Such theories can also be UV fundamental, yet they are not scale invariant. On the other hand, scale invariance seems to play some fundamental role in the construction of the quantum gravity theory(ies), see [6-9], so in this article we restrict to the fixed point case. The fixed point can be at zero (Gaussian fixed point), making the theory asymptotically free. Alternatively, it can reach some nonzero value (non-Gaussian fixed point/residual interaction). We call such theory asymptotically safe. Steven Weinberg hypothesized that gravity possesses an interacting fixed point $[10,11]$. This issue was studied in [12-14], where the calculations were done by means of $\epsilon$ expansion in the vicinity of two dimensions. However, in general, such fixed points cannot be considered by means of ordinary perturbation theory, where the theory is expanded around free theory, hence around the fixed point at zero values of the couplings. The study of such fixed points requires other, nonperturbative treatment.

The functional renormalization group (FRG) is one of the tools which can be used. In the FRG approach, one studies the evolution of the effective average action $\Gamma_{k}$, which is a quantum effective action, where all the interactions with momenta lower than $k$ are integrated out. The $\Gamma_{k}$ interpolates between the classical action $S_{\Lambda}$ at the UV scale $\Lambda$ and the full quantum effective action $\Gamma=\Gamma_{k=0}$. The evolution of $\Gamma_{k}$ is given by the Wetterich equation [15-17]. Using this approach, the gravitational fixed points were 
found (for Euclidean signature); see [18,19]. Moreover, the gravitational corrections to the matter beta functions can be calculated and they alter the UV running of the matter couplings. Despite the fact that the asymptotic safety program for quantum gravity is far from being finished, see $[20,21]$, yet it seems to be a very promising way to quantize gravity, not only because of its simplicity, but also due to its rich particle physics phenomenology, which can be tested. In particular, two years before the discovery of the Higgs boson, its mass was calculated in [22] as $126 \pm$ few GeV. However, the authors took the top quark mass smaller than the current observed value. In this article, we repeat this calculation and investigate the possible sources of disparity between current experimental measurements and the theoretical predictions.

However, the asymptotic safety program has more predictions in case of particle physics. For example, since the top Yukawa coupling is close to the upper bound in the basin of attraction, hence if it runs to the interacting fixed point, then it is also predictable [23]. In such scenario, the difference between the top and the bottom quark masses [24] can also be predicted. Moreover, the fine structure constant in grand unified theories can also be predicted [25]. These results are promising; however, the results for the Higgs mass calculated for the top interacting fixed point scenario [23] give $m_{H} \approx 132 \mathrm{GeV}$. The authors stress that the results arise in a truncation of the RG flow that is limited to the surmised leading-order effects of quantum gravity on matter [23]. This might be the case, see [26-28], however the Planck suppressed couplings did not affect the running in the IR [29,30]. The $\lambda(\mu)$ becomes negative at $10^{10} \mathrm{GeV}$, and in this article, we explore another possibility, namely that addition of beyond standard model fields results in the correct predictions for the Higgs mass. The fact that most of the problems of the standard model can be solved at $\sim 1 \mathrm{TeV}$ scale [31-34] supports that view and the new physics should affect the prediction of the Higgs mass. In particular, we analyze two scenarios: addition of the $Z^{\prime}$ boson, which is related to the $\mathrm{B}$ anomalies, and addition of sterile quarks.

\section{CALCULATION OF THE HIGGS BOSON MASS IN THE STANDARD MODEL}

In this paragraph, we reevaluate the calculations done in [22] concerning the calculation of the Higgs mass. The Higgs part of standard model Lagrangian is given by

$$
\mathcal{L}_{\text {Higgs }}=\left(D_{\mu} H^{\dagger} D^{\mu} H\right)-\lambda\left(\left(H^{\dagger} H\right)^{2}-v^{2}\right)^{2},
$$

where $v_{H} \approx 246.22 \mathrm{GeV}$. On the tree level, one has

$$
m_{H}^{2}=2 \lambda v^{2},
$$

and the radiative corrections are $\mathcal{O}(1) \mathrm{GeV}$. The one-loop beta functions (where $\hat{\beta}_{\mathrm{SM}}=16 \pi^{2} \beta_{\mathrm{SM}}$ ) in the $\overline{\mathrm{MS}}$ scheme are

$$
\begin{aligned}
\hat{\beta}_{g_{1}}= & \frac{41}{6} g_{1}^{3}, \quad \hat{\beta}_{g_{2}}=-\frac{19}{6} g_{2}^{3}, \quad \hat{\beta}_{g_{3}}=-7 g_{3}^{3}, \\
\hat{\beta}_{y_{t}}= & y_{t}\left(\frac{9}{2} y_{t}^{2}-8 g_{3}^{2}-\frac{9}{4} g_{2}^{2}-\frac{17}{12} g_{1}^{2}\right), \\
\hat{\beta}_{\lambda_{1}}= & 24 \lambda_{1}^{2}-3 \lambda_{1}\left(3 g_{2}^{2}+g_{1}^{2}-4 y_{t}^{2}\right) \\
& +\frac{9}{8} g_{2}^{4}+\frac{3}{4} g_{2}^{2} g_{1}^{2}+\frac{3}{8} g_{1}^{4}-6 y_{t}^{4},
\end{aligned}
$$

where $g_{1}, g_{2}, g_{3}$ are $U(1), S U(2), S U(3)$ standard model gauge couplings, respectively, and $y_{t}$ is the top Yukawa coupling. The two-loop beta functions, we have used in our calculations, are given in $[35,36]$. The gravitational corrections $[22,37-39]$ to the beta functions are in the leading order

$$
\beta_{i}^{\text {grav }}\left(g_{i}, \mu\right)=\frac{a_{i}}{8 \pi} \frac{\mu^{2}}{M_{P}^{2}+2 \xi_{0} \mu^{2}} g_{i},
$$

where $M_{P}=2.4 \times 10^{18} \mathrm{GeV}$ is the low energy Planck mass, $\xi_{0}$ is related to the gravitational fixed point and depends on the matter content; see Eq. (8). For the standard model, one has $\xi_{0} \approx 0.024$ and $a_{\lambda}=+3, \quad a_{y_{t}}=-0.5, \quad a_{g_{i}}=-1$. Depending on the sign of $a_{i}$, one gets repelling/attracting fixed point at zero for a given coupling. If one demands that all of the matter couplings to be asymptotically free, then the ones with the repelling fixed points become predictable and the ones with attracting fixed points have to be inside the basin of attraction, otherwise they will diverge [22]. Since $a_{\lambda}=+3$, then Higgs self-coupling has a repelling fixed point at zero and becomes a prediction of a theory rather than being a free parameter. On the two-loop level and for $y_{t}=g_{1}=g_{2}=g_{3}=0$, one has

$\beta_{\lambda}(\mu)=\frac{1}{16 \pi^{2}}\left(24 \lambda^{2}-\frac{312}{16 \pi^{2}} \lambda^{3}\right)+\frac{a_{\lambda}}{8 \pi} \frac{\mu^{2}}{M_{P}^{2}+2 \xi_{0} \mu^{2}} \lambda$,

which has the following fixed points: $\lambda=0$ (repeller), $\lambda \approx 21$ (attractor), $\lambda \approx-9.36$ (attractor). For this reason, two basins of attraction are separated by the single trajectory going to the repelling fixed point. The numerical calculations confirm that if at any scale below Planck scale $\lambda(\mu)<0$, then it drops to the nonperturbative fixed point. If one assumes that $\lambda$ has to stay in the perturbative region, then necessarily one gets $\lambda(\mu) \geq 0$ at all scales. Furthermore, in order to avoid the attractor in the positive domain, one should assume that (again confirmed by numerics at two-loop level)

$\lambda=\min \left\{\bar{\lambda}: \forall_{\mu} \bar{\lambda}(\mu) \geq 0, \bar{\lambda}\left(M_{P}\right) \approx 0 \quad\right.$ and $\left.\beta_{\bar{\lambda}},\left(M_{P}\right) \approx 0\right\}$,

which agrees with the arguments of the authors of [22]. Then one needs stable electroweak (EW) vacuum in order to predict the Higgs mass in the line of [22]. This reasoning explains also the multiple point principle postulated in [40]. According to this principle, there are two vacuum states with 
about the same energy density, one at electroweak scale and one at the Planck scale, which can be used to predict the SM couplings [40-42]. It has also interesting cosmological consequences [43]. Let us note that the requirement, Eq. (7), is actually stronger than the EW stability, since the stability can be affected by Planck suppressed operators, while positivity of $\lambda$ is not affected by Planck physics $[29,30]$.

The current calculations of running of $\lambda(\mu)$ show that $\lambda(\mu)$ drops to negative values at roughly $10^{10} \mathrm{GeV}[44,45]$, making the vacuum metastable. Also, the situation is similar if one takes into account the nonminimal $H^{\dagger} H R$ term [46]. However, it was suggested in [47] that our vacuum can be stable for some space of parameters. Yet for central values of $m_{\text {top }}$ and $m_{H}$ the vacuum is metastable with the estimation of the lower stability bound is $m_{H}>$ $(129.6 \pm 1.5) \mathrm{GeV}[44,45]$. On the other hand, from the experimental point of view, the Higgs mass is constrained as $m_{H}=125.18 \pm 0.18 \mathrm{GeV}$ [48], which corresponds to $\lambda\left(m_{\text {top }}\right)=0.127823 \pm 0.000367$ in $\overline{\mathrm{MS}}$ scheme for oneloop matching conditions $[35,36,49]$ (and $\lambda=0.12924 \pm$ 0.00037 at the tree level), where we have taken into account the uncertainties in the measurements of the top quark $m_{\text {top }}=173.0 \pm 0.4$ [48]. Hence, the stability of EW vacuum, assumed in [22], is in contradistinction with the measured Higgs value. Yet this stability bound is close enough to the experimental value of the Higgs mass, that one can hope that a slight extension of the SM can bring it to the correct value.

To obtain the predictions for $\lambda$, we do the two-loop running of the $g_{1}, g_{2}, g_{3}, y_{t}, \lambda$ with gravitational corrections and search for optimal $\lambda$ for given set of $g_{1}, g_{2}, g_{3}, y_{t}$, such that $\lambda \geq 0$ and there are no Landau poles ( $\lambda$ does not end in the nonperturbative region). Then given $\lambda$ one can recover the Higgs mass via matching relations (let us note that we treat $v$ as given from experiment).

In our analysis, we take one-loop matched parameters as [44] $g_{1}\left(m_{\text {top }}\right)=0.35940, g_{2}\left(m_{\text {top }}\right)=0.64754, g_{3}\left(m_{\text {top }}\right)=$ 1.18823 , and we scan over one-loop matched $y_{t}$ for various experimentally viable $m_{\text {top }}$, giving $y_{\text {top }}\left(m_{\text {top }}\right)=$ $0.94759 \pm 0.0022$, which is slightly lower than the central value obtained in [44]. As a result, we get $\lambda=0.15102 \pm$ 0.00158 giving $m_{H} \approx 135 \mathrm{GeV}$ at one loop and $\lambda=$ $0.13866 \pm 0.00218$ at two loops (the uncertainties are due to the $y_{t}$ coupling) and $m_{H} \approx 130.5 \mathrm{GeV}$. Actually, the two-loop result is close to the stability bound of the Higgs mass, which means high degree of accuracy.

We have checked that if one takes the bottom quark and the taon into account, it changes the predictions for $m_{H}$ less than $1 \mathrm{KeV}$, which is far below the theoretical and experimental accuracy. This can be expected since $y_{b}\left(m_{\text {top }}\right) \approx 0.015$ [50]. Due to metastability of vacuum, we see that it is necessary to introduce the beyond standard model operators in order for Higgs mass to be predicted in the asymptotic safety paradigm at the correct experimental value.

\section{BEYOND STANDARD MODEL}

\section{A. Gravitation constraints}

First, let us constrain the possible additional matter content. We have

$$
\xi_{0}=\frac{1}{16 \pi G_{N}^{*}} \text { and } G_{N}^{*} \approx-\frac{12 \pi}{N_{S}+2 N_{D}-3 N_{V}-46},
$$

where $N_{S}, N_{D}, N_{V}$ are the number of scalars, fermions, and vector particles, respectively. We know that $G_{N}\left(m_{\text {top }}\right) \geq 0$ and the running of $G_{N}$ cannot change the sign of $G_{N}$ [18]. Then we have $G_{N}^{*} \geq 0$. So the beyond standard model theories which extend the standard model broadly can be incompatible with the asymptotic safety paradigm. For example, such theories are minimal supersymmetric standard model [51] and some of the grand unified theories $[52,53]$. It seems $[21,54]$ that asymptotic safety prefers the minimal extensions of the standard model. One should note that these effects can possibly be truncations artefacts and can disappear after taking into account more operators. On the other hand, the actual change of $\xi_{0}$ due to small modifications of the SM does not alter the predictions at observable level.

\section{B. Models}

As we have said the prediction of $\lambda$ depends highly on the initial value of top Yukawa coupling. It also strongly depends on the running of $y_{t}$. So, changing this running alters the prediction of $\lambda$ from asymptotic safety. In this paragraph, we shall discuss two extensions of the standard model, where $\beta_{y_{t}}$ is slightly changed, because to predict the correct value of $\lambda$ it seems that only a minor effect is required. In both models, we extend the Higgs sector by an additional complex scalar singlet under $S U(3)_{c}$, $S U(2)_{L} \times U(1)_{Y}$ gauge groups [55],

$$
\begin{aligned}
\mathcal{L}_{\text {scalar }}= & \left(D_{\mu} H\right)^{\dagger}\left(D^{\mu} H\right)+\left(\partial_{\mu} \phi^{*} \partial^{\mu} \phi\right)-V(H, \phi), \\
V(H, \phi)= & -m_{1}^{2} H^{\dagger} H-m_{2}^{2} \phi^{\star} \phi+\lambda_{1}\left(H^{\dagger} H\right)^{2} \\
& +\lambda_{2}\left(\phi^{\star} \phi\right)^{2}+2 \lambda_{3}\left(H^{\dagger} H\right) \phi^{\star} \phi .
\end{aligned}
$$

Often one also includes right-handed neutrinos coupled to $\phi[32,56,57]$, yet they would not be relevant to our discussion. The inclusion of portal interaction stabilizes the vacuum [1,32] (also with inclusion of higher order operators [58]), yet in our further analysis we shall put $\lambda_{3}=0$. So there will be no portal stabilization effect. This is in line with the asymptotic safety analysis of such models [59], where one needs $\lambda_{3}=0$ at all scales.

\section{Model I}

In Model I, the global, not anomalous SM group $U(1)_{B-L}$, related to the baryon minus lepton $(B-L)$ number, is gauged and a new Gauge boson $B_{\mu}^{\prime}$ [60-62] 
is introduced. Then the covariant derivatives receive an additional contribution $D_{\mu} \rightarrow D_{\mu}+i\left(\tilde{g} Y+g_{1}^{\prime} Y_{B-L}\right) B_{\mu}^{\prime}$, where $Y$ is the hypercharge and $Y_{B-L}$ is the (B-L)-charge. The $B_{\mu}^{\prime}$ boson becomes massive due to the nonzero vacuum expectation value of $\phi$, and the $\tilde{g}$ describes the mixing between $Z$ and $Z^{\prime}$ after spontaneous symmetry breaking. Following [62], we analyze the "pure" $B-L$ model by assuming that there is no tree level mixing between $\mathrm{Z}$ bosons $\left(\tilde{g}\left(m_{\text {top }}\right)=0\right)$, which is supported by the current data [48], which might be spoiled by radiative corrections. This model is also supported experimentally, since it is the most popular way of explaining the so-called $B$ anomalies [63-66], which are the observed inconsistencies of the SM with experimental data in the bottom quark decays. The new terms in the beta functions at the one-loop level are (for $\tilde{g}=0$ )

$$
\hat{\beta}_{g_{1}^{\prime}}=12 g_{1}^{\prime 3}, \quad \hat{\beta}_{y_{t}}=\hat{\beta}_{y_{t}}^{\mathrm{SM}}-\frac{2}{3} y_{t} g_{1}^{\prime 2} \theta\left(\mu-M_{Z^{\prime}}\right) .
$$

The mass of the $Z^{\prime}$ boson is restricted to be $\frac{M_{Z^{\prime}}}{g_{1}^{\prime}}>7 \mathrm{TeV}$ or $M_{Z^{\prime}} \leq 2 m_{\text {top }}$ [67].

\section{Model II}

The Model II is inspired by Kim-Shifman-VainshteinZakharov axion $[68,69]$ and includes new sterile (EW singlet) quarks $Q_{i}$ charged under $U(1)_{P Q}$ coupled to new scalar,

$$
\begin{aligned}
\mathcal{L}= & \mathcal{L}_{\text {fermions }}+\mathcal{L}_{\mathrm{Y}}+\mathcal{L}_{\text {gauge }}+\mathcal{L}_{\text {scalar }} \\
& +\sum_{i=1}^{n}\left(\bar{Q}_{i} D_{\mu} \gamma^{\mu} Q_{i}-y_{Q} \phi \bar{Q}_{i} Q_{i}+\text { H.c. }\right),
\end{aligned}
$$

where we assume that Yukawa matrix $\mathbf{y}_{Q}$ to be diagonal and the quarks acquire masses $M_{i}=y_{Q} v_{\phi} / \sqrt{2}$. The "phase" of $\phi$ is called the axion particle and becomes massive due to instanton effects. This model was proposed to solve the strong charge parity $(C P)$ problem [70] by spontaneous symmetry breaking of $U(1)_{P Q}$ [71]. As a side comment let us note that asymptotic safety gives a possible explanation to the strong $C P$ problem without axions. The strong $C P$ violation consists of two terms $\theta_{\mathrm{QCD}}=\theta_{\text {topological }}+$ $\arg \operatorname{det} M_{u} M_{d}$ and in principle $\arg \operatorname{det} M_{u} M_{d}$ should give much bigger contribution to the strong $C P$ violation. By considering the gravitational corrections, the following reasoning can, at least partially, explain the smallness of the strong $C P$-violation effect. Namely, in the case of $\arg \operatorname{det} M_{u} M_{d}$ there is no running till at least seven loops [70]. Despite the fact that the gravitational corrections Eq. (5) are extremely small, yet they can overtake the dynamics even in the IR and drop $\arg \operatorname{det} M_{u} M_{d}$ to zero, since the matter contributions are $\mathcal{O}\left(\left(\arg \operatorname{det} M_{u} M_{d}\right)^{17}\right)$. In order for gravitational contributions to be dominant, one needs $0.01 \gtrsim \arg \operatorname{det} M_{u} M_{d}$, which is far beyond the experimental bounds. However, this argument requires a more detailed analysis.

Even with $\lambda_{3}=0$, the running of $g_{3}$ is affected by the inclusion of the heavy quarks,

$16 \pi^{2} \frac{d g_{3}}{d \log \mu}=\hat{\beta}\left(g_{3}\right) \rightarrow \hat{\beta}\left(g_{3}\right)+\frac{2}{3} \sum_{i=1}^{n} \theta\left(\mu-M_{Q_{i}}\right) g_{3}^{3}$,

which in turn alters the running of $y_{t}$. Let us note that if there are many such quarks, then even the asymptotic freedom of the QCD can be spoiled. However, in our analysis, we focus on addition of one or two sterile quarks into the SM.

\section{Calculations}

At first let us note that both models agree with the condition given by Eq. (8), with $\xi_{1}=0.02$ and $\xi_{2}=0.023$ accordingly. Asymptotic safety requirement gives restrictions on the couplings of new degrees of freedom (d.o.f.). In case of Model I, one gets that $g_{1}^{\prime}\left(m_{\text {top }}\right) \in[0.0,0.4]$ (with $\left.a_{g_{1}^{\prime}}, a_{\tilde{g}}=-1\right)$. On the other hand, the minimal mass for the sterile quark from Model II is $m_{Q} \sim 100 \mathrm{TeV}$, otherwise the running becomes unstable. Furthermore, if one includes one more quark, then its mass is of the order of $10^{6} \mathrm{TeV}$ giving a huge hierarchy, which seems to be very unnatural. Since then we shall restrict ourselves to one heavy, sterile quark. Below, in Fig. 1, we present the calculations for Model I using the two-loop beta functions for the couplings; see [72-74].

As we can see on the plot for large $g_{1}^{\prime}$ and small $M_{Z^{\prime}}$, the Higgs mass is getting close to the experimental value. For $y_{t}=0.9539$, one even gets $m_{H} \approx 126 \mathrm{GeV}$. If we use more precise formulas for two-loop matching [44], then we get that the central value $y_{t} \approx 0.94$. For this central value and for $g_{1}^{\prime}>0.3, m_{Z^{\prime}}<2 m_{\text {top }}$ Higgs mass is $m_{H}=125 \pm$ $1.5 \mathrm{GeV}$ depending on the exact parameters. In Fig. 2, we show this dependence using two-loop matched $y_{t}=0.94$ and two-loop matched $y_{t}=0.936$ with higher order QCD corrections.

The Model I satisfies criteria of EW vacuum stability and is of experimental and theoretical interest. The asymptotic

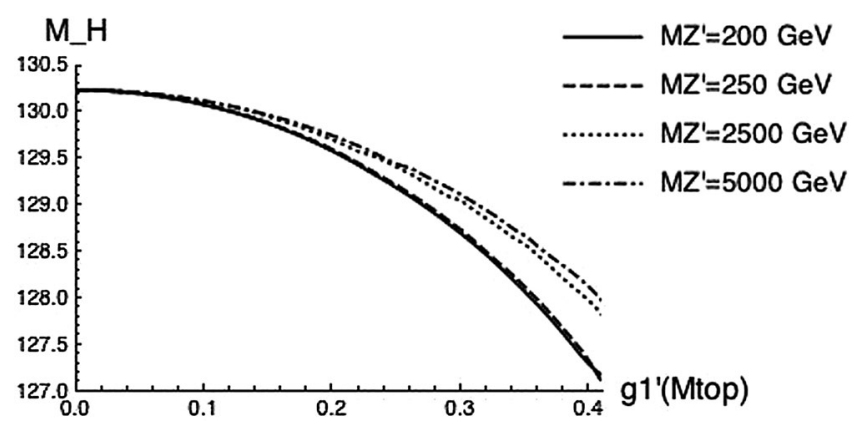

FIG. 1. Higgs mass for various $g_{1}^{\prime}$ and $M_{Z^{\prime}}, y_{t}=0.94759$. 


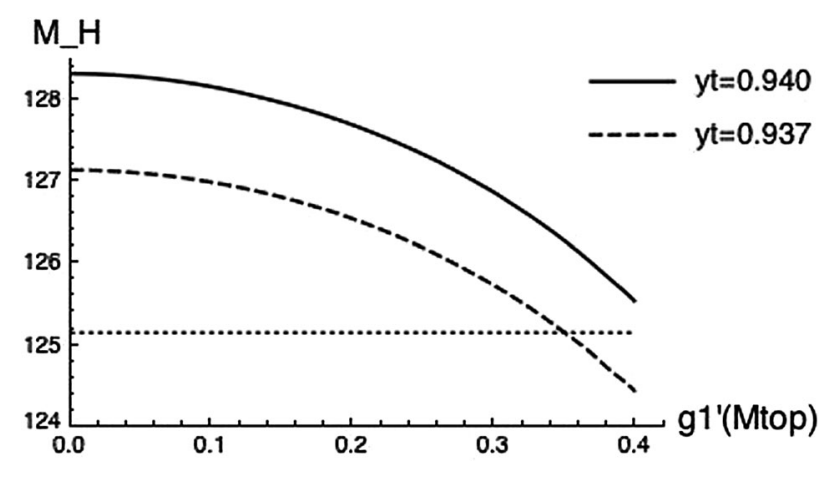

FIG. 2. Higgs mass for $M_{Z^{\prime}}=200 \mathrm{GeV}$, higher loop matching.

safety predicts that the mass of the new gauge boson should be small, which can be verified experimentally. Furthermore, our argument is confirmed by the fact that for certain space of parameters, the EW vacuum in the B-L extension is stable [72,75-77]. The effect of introducing $Z^{\prime}$ boson can be even more significant if the Higgs boson is also charged under $U_{B-L}$; see [75]. Yet in such models $Z^{\prime}$ is highly constrained observationally with $M_{Z^{\prime}}>3 \mathrm{TeV}$. Moreover, if one relaxes the condition $\lambda_{3}(\mu)=0$, which is the case in more general $B-L$ models, then one immediately gets the stability of EW vacuum $[32,78,79]$ and hence correct Higgs mass.

Furthermore, we have checked that the $\tilde{g}$ corrections and inclusion of right-handed neutrinos, bottom quark, and taon give the contributions which are negligible. For instance, taking into account the $y_{N}$ right-handed neutrino Yukawa coupling two-loop contribution [72] gives the difference of $10 \mathrm{MeV}$ between $y_{N}=0.0$ and $y_{N}=0.44$ situation.

In the case of Model II, we perform the full two-loop analysis, with one quark $Q$ and with masses in range $m_{Q} \in\left(10^{5}-10^{18}\right) \mathrm{GeV}$. The results are shown below in Fig. 3.

For Model II, the new d.o.f. influence the running of $\lambda$ much less than in Model I. The change in predicted Higgs mass at the one-loop matching is of order $1.5 \mathrm{GeV}$ downward. There are two reasons for that. First of all, addition of $Q$ changes only running of $g_{3}$, which in turn changes the running of $y_{t}$ and has only slight effect on $\lambda$. Second, new

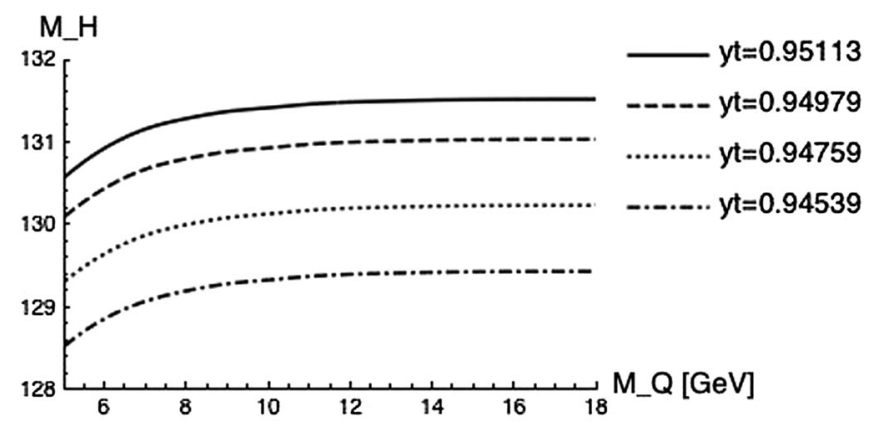

FIG. 3. Optimized $\lambda\left(m_{\text {top }}\right)$ for various $M_{Q}$ (in logarithmic scale) and $y_{t}$. d.o.f. are constrained to have mass far beyond the EW scale, while the $Z^{\prime}$ mass is not constrained that much both theoretically and observationally. We can conclude that inclusion of additional sterile quarks cannot drop the Higgs mass to the correct value. Yet maybe Model II combined with Model I can give the correct Higgs mass.

\section{DISCUSSION}

There are a few issues which require separate discussion. First of all, to obtain the running of the considered couplings, one can solve the full Wetterich equation; see, for example, [23,80-82]. While Wetterich equation is exact, however it is very difficult (or even impossible) to be solved, because one has to take into account all of the operators which coincide with the symmetries. Moreover, one has to choose the cutoff, which is arbitrary $[21,83]$. So, in order to reproduce the correct perturbative results, one has to take into account many higher order operators and choose the proper cutoff. As a state of the art, the current FRG calculations match the usual results at the one-loop level and the leading contributions to running at two-loop level [82] for pure gauge theories. Moreover, the gravitational corrections are ambiguous due to gauge dependence, for example, the prediction of top mass ranges from 130 to $171 \mathrm{GeV}$ only due to this effect [23]. For the sake of phenomenology, we decided to use the loop expansion and the effective field theory gravitational corrections [37] supplemented with the gravitational fixed point calculated with the FRG techniques [54]. Furthermore, it seems that these two approaches give similar results (compare the fixed point of top Yukawa coupling in [22] and [23]).

One can also argue that $a_{i}[22,37,84]$ are not calculated to high accuracy, making the whole calculation very sensitive to those parameters, hence not reliable. This is indeed the case for non-Gaussian fixed point making the prediction of upper bound for top quark mass sensitive to new physics [23]. Yet in the case of Gaussian fixed point the existence of attractive/repelling fixed point at zero is much more vital than actual value of $a_{\lambda}$ due to the stability argument.

In our analysis, we use the $\overline{\mathrm{MS}}$ beta functions and parameters. For this scheme, we cannot use the AppelquistCarazzone theorem [85] and we rely on the effective field theory approach [86]. In this approach, one has to take into account the threshold effects $[87,88]$. In case of Model II, these threshold effects at two-loop precision change the prediction of Higgs mass by $5 \mathrm{MeV}$, which is far below both experimental and theoretical accuracy. On the other hand, for Model I, due to relatively small mass of $Z^{\prime}$, one should fit the observables to the new set of $\overline{\mathrm{MS}}$ couplings and then do the running; see discussion in [61]. Otherwise one can use mixed on-shell/MS scheme [61]. Both procedures are beyond the scope of this article and are left for future work. As a matter of fact, these theoretical uncertainties are superseded by much bigger experimental ones 
in the top and Higgs mass measurements (the fact that $g_{1}^{\prime}$ is relevant parameter also contributes to this uncertainty).

Finally, the $\xi_{0}$ depends not only on the matter content, but also on the gravity sector. For example, in unimodular gravity [89], it has slightly different value, yet the effect on Higgs mass is negligible [in naive calculations, one gets $\mathcal{O}(1 \mathrm{MeV})]$, yet it might be interesting to test it in the future. On the other hand, there are other more fundamental modifications of gravity, like massive gravity [90] or Horava gravity [91], and their fixed point structure might be completely different. Then with the right theoretical and experiment accuracy one can test quantum structure of spacetime in particle colliders far below Planck scale.

\section{CONCLUSIONS}

In this article, we have recalculated the Higgs mass in the standard model by taking into account the gravitational corrections and asymptotic safety requirements using the current observational bounds on $m_{\text {top }}$. Due to the stability bound, the Higgs mass is predicted to be a higher than the experimental value.

We have investigated the two beyond SM models which improve the running of $\lambda$. In Model I, we observed that with $\lambda_{3}=0$ one gets $m_{H} \approx 125 \mathrm{GeV}$ as the lowest value, which agrees with the stability bound. However, the other $Z^{\prime}$ models can give different predictions. The correct Higgs mass can also be obtained in the conformal standard model [78], where a new scalar d.o.f. is also constrained to have $m_{\phi} \approx 300 \mathrm{GeV}$. On the other hand, we have excluded the possibility that the addition of sterile quarks gives the correct $m_{H}$.

Our analysis shows that the addition of new d.o.f. can stabilize the electroweak vacuum at the experimental value of the Higgs Boson mass. One should stress once again that whole reasoning relies on the precise measurement of the top quark mass [92], and hence the conclusions can be altered by future measurements.

\section{ACKNOWLEDGMENTS}

J. H. K. thanks Piotr Chankowski, Frederic Grabowski, Krzysztof Meissner, and Mikhail Shaposhnikov for valuable and inspiring discussions and for comments on the first version of the article. J.H.K. also thanks anonymous referee for careful reading of the article and constructive critique which resulted in substantially improved article. J.H.K. would like to acknowledge the Max Planck Institute for Gravitational Physics (Albert Einstein Institute) hospitality and support during this work. J. H. K. was partially supported by the Polish National Science Center Grant No. DEC-2017/25/B/ST2/00165.
[1] K. A. Meissner and H. Nicolai, Phys. Lett. B 648, 312 (2007).

[2] S. Coleman and E. Weinberg, Phys. Rev. D 7, 1888 (1973).

[3] S. Gukov, Nucl. Phys. B919, 583 (2017).

[4] S. M. Dawid, R. Gonsior, J. H. Kwapisz, K. Serafin, M. Tobolski, and S. Głazek, Phys. Lett. B 777, 260 (2018).

[5] S. Moroz and R. Schmidt, Ann. Phys. (Amsterdam) 325, 491 (2010).

[6] G. t Hooft, Int. J. Mod. Phys. D 24, 1543001 (2015).

[7] G. t Hooft, in Proceedings, 14th Marcel Grossmann Meeting on Recent Developments in Theoretical and Experimental General Relativity, Astrophysics, and Relativistic Field Theories (MG14) (In 4 Volumes): Rome, Italy, 2015 (World Scientific, Singapore, 2017), Vol. 1, pp. 3-12.

[8] L. Rachwał, Universe 4, 125 (2018).

[9] C. Wetterich, arXiv:1901.04741.

[10] S. Weinberg, Critical phenomena for field theorists, Lectures of Subnuclear Physics, Ettore Majorana, Erice, Sicily (1976), p. 1.

[11] S. Weinberg, in General Relativity: An Einstein Centenary Survey, edited by S. W. Hawking and W. Israel (Cambridge University Press, Cambridge, 1979), pp. 790-831.

[12] R. Gastmans, R. Kallosh, and C. Truffin, Nucl. Phys. B133, 417 (1978).

[13] S. M. Christensen and M. J. Duff, Phys. Lett. 79B, 213 (1978).
[14] L. Smolin, Nucl. Phys. B208, 439 (1982).

[15] C. Wetterich, Phys. Lett. B 301, 90 (1993).

[16] T. R. Morris, Int. J. Mod. Phys. A 09, 2411 (1994).

[17] T. R. Morris, Phys. Lett. B 329, 241 (1994).

[18] M. Reuter, Phys. Rev. D 57, 971 (1998).

[19] D. Dou and R. Percacci, Classical Quantum Gravity 15, 3449 (1998).

[20] A. Eichhorn, Front. Astron. Space Sci. 5, 47 (2019).

[21] R. Percacci, An Introduction to Covariant Quantum Gravity and Asymptotic Safety (World Scientific, Singapore, 2017).

[22] M. Shaposhnikov and C. Wetterich, Phys. Lett. B 683, 196 (2010).

[23] A. Eichhorn and A. Held, Phys. Lett. B 777, 217 (2018).

[24] A. Eichhorn and M. Schiffer, Phys. Lett. B 793, 383 (2019).

[25] A. Eichhorn, A. Held, and C. Wetterich, Phys. Lett. B 782, 198 (2018).

[26] H. Gies, R. Sondenheimer, and M. Warschinke, Eur. Phys. J. C 77, 743 (2017).

[27] A. Eichhorn, H. Gies, J. Jaeckel, T. Plehn, M. M. Scherer, and R. Sondenheimer, J. High Energy Phys. 04 (2015) 022.

[28] F. Loebbert and J. Plefka, Mod. Phys. Lett. A 30, 1550189 (2015).

[29] V. Branchina and E. Messina, Phys. Rev. Lett. 111, 241801 (2013).

[30] V. Branchina, E. Messina, and A. Platania, J. High Energy Phys. 09 (2014) 182. 
[31] M. Shaposhnikov, in Astroparticle Physics: Current Issues, 2007 (APCI07) Budapest, Hungary (2007), https://arxiv .org/abs/0708.3550.

[32] A. Lewandowski, K. A. Meissner, and H. Nicolai, Phys. Rev. D 97, 035024 (2018).

[33] P. H. Chankowski, A. Lewandowski, K. A. Meissner, and H. Nicolai, Mod. Phys. Lett. A 30, 1550006 (2015).

[34] K. A. Meissner, H. Nicolai, and J. Plefka, Phys. Lett. B 791, 62 (2019).

[35] M. E. Machacek and M. Vaughn, Nucl. Phys. B249, 70 (1985).

[36] H. Arason, D. J. Castano, B. Kesthelyi, S. Mikaelian, E. J. Piard, P. Ramond, and B. D. Wright, Phys. Rev. D 46, 3945 (1992).

[37] S. P. Robinson and F. Wilczek, Phys. Rev. Lett. 96, 231601 (2006).

[38] A. Eichhorn, Y. Hamada, J. Lumma, and M. Yamada, Phys. Rev. D 97, 086004 (2018).

[39] J. M. Pawlowski, M. Reichert, C. Wetterich, and M. Yamada, Phys. Rev. D 99, 086010 (2019).

[40] D. L. Bennett and H. B. Nielsen, Int. J. Mod. Phys. A 09, 5155 (1994).

[41] C. D. Froggatt and H. B. Nielsen, Phys. Lett. B 368, 96 (1996).

[42] C. D. Froggatt and H. B. Nielsen, Surveys High Energy Phys. 18, 55 (2003).

[43] B. G. Sidharth, C. R. Das, C. D. Froggatt, H. B. Nielsen, and L. Laperashvili, arXiv:1801.06979.

[44] D. Buttazzo, G. Degrassi, P. P. Giardino, G. F. Giudice, F. Sala, A. Salvio, and A. Strumia, J. High Energy Phys. 12 (2013) 089.

[45] F. Bezrukov, M. Y. Kalmykov, B. A. Kniehl, and M. Shaposhnikov, J. High Energy Phys. 10 (2012) 140.

[46] F. Bezrukov, J. Rubio, and M. Shaposhnikov, Phys. Rev. D 92, 083512 (2015).

[47] A. V. Bednyakov, B. A. Kniehl, A. F. Pikelner, and O. L. Veretin, Phys. Rev. Lett. 115, 201802 (2015).

[48] M. Tanabashi et al. (Particle Data Group), Phys. Rev. D 98 , 030001 (2018).

[49] A. Sirlin and R. Zucchini, Nucl. Phys. B266, 389 (1986).

[50] A. V. Bednyakov, B. A. Kniehl, A. F. Pikelner, and O. L. Veretin, Nucl. Phys. B916, 463 (2017).

[51] S. Dimopoulos, S. Raby, and F. Wilczek, Phys. Rev. D 24, 1681 (1981).

[52] A. Buras, J. Ellis, M. Gaillard, and D. Nanopoulos, Nuc. Phys. B135, 66 (1978).

[53] L. Ibáñez and G. Ross, Phys. Lett. B 105, 439 (1981).

[54] C. Wetterich and M. Yamada, Phys. Rev. D 100, 066017 (2019).

[55] B. Patt and F. Wilczek, arXiv:hep-ph/0605188.

[56] M. Shaposhnikov and I. Tkachev, Phys. Lett. B 639, 414 (2006).

[57] M. Drewes, Int. J. Mod. Phys. E 22, 1330019 (2013).

[58] A. Eichhorn and M. M. Scherer, Phys. Rev. D 90, 025023 (2014).

[59] A. Eichhorn, Y. Hamada, J. Lumma, and M. Yamada, Phys. Rev. D 97, 086004 (2018).
[60] P. Langacker, Rev. Mod. Phys. 81, 1199 (2009).

[61] P. H. Chankowski, S. Pokorski, and J. Wagner, Eur. Phys. J. C 47, 187 (2006).

[62] L. Basso, Phenomenology of the minimal B-L extension of the standard model at the LHC, Ph.D. thesis, Southampton University, 2011.

[63] R. Aaij et al. (LHCb Collaboration), J. High Energy Phys. 02 (2016) 104.

[64] R. Aaij et al. (LHCb Collaboration), J. High Energy Phys. 08 (2017) 055.

[65] A. D. Sierra, F. Staub, and A. Vicente, Phys. Rev. D 92, 015001 (2015).

[66] W. Altmannshofer and D. M. Straub, Eur. Phys. J. C 75, 382 (2015).

[67] C.-W. Chiang, T. Nomura, and K. Yagyu, J. High Energy Phys. 05 (2014) 106.

[68] J. E. Kim, Phys. Rev. Lett. 43, 103 (1979).

[69] M. A. Shifman, A. Vainshtein, and V. I. Zakharov, Nucl. Phys. B166, 493 (1980).

[70] J. R. Ellis and M. K. Gaillard, Nucl. Phys. B150, 141 (1979).

[71] R. D. Peccei and H. R. Quinn, Phys. Rev. Lett. 38, 1440 (1977).

[72] A. Das, N. Okada, and N. Papapietro, Eur. Phys. J. C 77, 122 (2017).

[73] F. del Aguila, G. D. Coughlan, and M. Quiros, Nucl. Phys. B307, 633 (1988); 312, 751(E) (1989).

[74] M.-x. Luo and Y. Xiao, Phys. Lett. B 555, 279 (2003).

[75] S. Di Chiara, V. Keus, and O. Lebedev, Phys. Lett. B 744, 59 (2015).

[76] C. Corianò, L. D. Rose, and C. Marzo, J. High Energy Phys. 02 (2016) 135

[77] Z.-W.Wang, F. S. Sage, T. Steele, and R. B. Mann, J. Phys. G 45, 095002 (2018).

[78] F. Grabowski, J. H. Kwapisz, and K. A. Meissner, Phys. Rev. D 99, 115029 (2019).

[79] A. Latosinski, A. Lewandowski, K. A. Meissner, and H. Nicolai, J. High Energy Phys. 10 (2015) 170.

[80] A. Eichhorn and A. Held, Phys. Rev. Lett. 121, 151302 (2018).

[81] A. Eichhorn, Found. Phys. 48, 1407 (2018).

[82] C. Wetterich, Nucl. Phys. B934, 265 (2018).

[83] D. F. Litim, Phys. Rev. D 64, 105007 (2001).

[84] O. Zanusso, L. Zambelli, G. P. Vacca, and R. Percacci, Phys. Lett. B 689, 90 (2010).

[85] T. Appelquist and J. Carazzone, Phys. Rev. D 11, 2856 (1975).

[86] S. Weinberg, Phys. Lett. 91B, 51 (1980).

[87] D. A. Ross, Nucl. Phys. B140, 1 (1978).

[88] L. J. Hall, Nucl. Phys. B178, 75 (1981).

[89] A. Eichhorn, Classical Quantum Gravity 30, 115016 (2013).

[90] C. de Rham, Living Rev. Relativity 17, 7 (2014).

[91] P. Horava, Phys. Rev. D 79, 084008 (2009).

[92] F. Bezrukov and M. Shaposhnikov, Zh. Eksp. Teor. Fiz. 147, 389 (2015) [J. Exp. Theor. Phys. 120, 335 (2015)]. 